\title{
Joining the pieces
}

Heterojunctions made of dissimilar semiconductors are at the heart of many modern technological marvels (for example, transistors). Now they can be downshifted to the nanoscale in a simple way: heterojunctions made of different types of graphene nanoribbons can be fabricated with atomic precision starting from a single precursor, as Michael Crommie and colleagues report in Nature Nanotechnology.

Cutting graphene into thin, atomically precise strips - graphene nanoribbons - endows graphene with potentially useful properties, such as a bandgap that depends on the width of the ribbon. Using bottom-up fabrication processes, in which molecular precursors react and combine on a surface to yield desired structures, it is possible to fabricate many different types of nanoribbons, depending on the starting molecule. "A very natural idea was to create heterojunctions in which the properties could change within a single ribbon, by building it from different molecular building blocks or by somehow modifying a single type of building block," explains Crommie. "Heterojunctions are extremely useful in electrical engineering applications, thus we decided to put

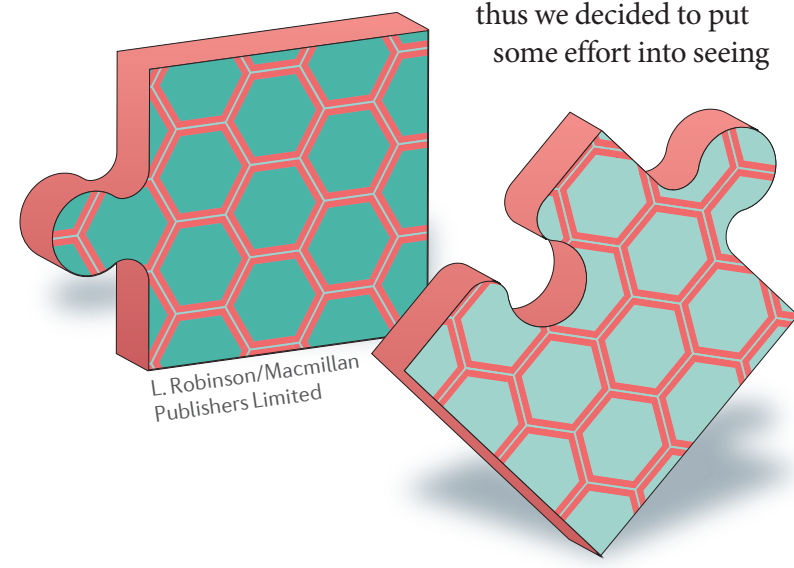

how small a heterojunction we could build and to understand how it might behave."

unfunctionalized
nanoribbons might now
be grown and positioned
in an initial layout, and
heterojunctions formed
afterwards by post-growth
techniques

Graphene nanoribbon heterojunctions are not new, but up until now they were obtained from random combinations of different precursor molecules, and they could not be modified after the synthetic process was completed. By contrast, Crommie and co-workers produced graphene nanoribbons by starting from a single precursor molecule (synthesized by Felix Fischer's group), which contains sacrificial carbonyl groups, and then functionalizing them post-synthesis. This was done through cleavage of the sacrificial groups by thermal annealing, which led to well-defined heterojunctions within individual nanoribbons. "The behaviour of devices this tiny can change dramatically if even a single atom is misplaced: we have taken advantage of this by selectively removing a few atoms to convert homogeneous nanoribbons into more useful heterojunction structures," comments Crommie.

The heterojunctions were characterized with scanning tunnelling microscopy and spectroscopy (STM/STS), but also with a new scanning probe technique, bond-resolved STM, which enables the imaging of chemical bonds at low temperature. Bond-resolved STM allows the observation of the carbonyl groups, which are not visible in conventional STM images, and thus the discrimination of functionalized from unfunctionalized nanoribbons, enabling the structure-property characterization of the heterostructures. These measurements revealed that the bandgap and energy alignment are different in functionalized and unfunctionalized nanoribbons, and that the electronic structure changes on an extremely short length scale generating very large effective fields within the heterojunction, as predicted by theoretical calculations.

One of the most interesting aspects of this synthetic route for graphene nanoribbons is that it allows the modification of their electronic properties and the formation of heterostructures after the growth process is completed. "In modern electronics, circuits are often built by first putting down a skeleton structure of device elements and then layering them with changes," says Crommie. "In the same way, unfunctionalized nanoribbons might now be grown and positioned in an initial layout, and heterojunctions formed afterwards by post-growth techniques, such as local heating or the application of other stimuli."

The next steps in this research include incorporating nanoribbon heterojunctions into electrical devices, testing their behaviour as an electrical current is passed through them, and understanding how this behaviour differs from that of more traditional, larger heterojunction devices.

Giulia Pacchioni

ORIGINAL ARTICLE Nguyen. G. D. et al. Atomically precise graphene nanoribbon heterojunctions from a single molecular precursor. Nat. Nanotechnol. http://dx.doi.org/10.1038/ nnano.2017.155 (2017) 